\title{
Precision Calibration of Large Area Micromegas Detectors Using Cosmic Muons
}

\author{
Philipp Lösel ${ }^{* a}$, Otmar Biebel ${ }^{a}$, Jonathan Bortfeldt ${ }^{b}$, Bernhard Flierl ${ }^{a}$, Maximilian \\ Herrmann $^{a}$, Ralf Hertenberger ${ }^{a}$, Felix Klitzner ${ }^{a}$, Ralph Müller ${ }^{a}$ and Andre Zibell ${ }^{c}$ \\ ${ }^{a}$ Ludwig-Maximilians-Universität München, Germany \\ ${ }^{b}$ CERN PH Department, Geneve, Switzerland \\ ${ }^{c}$ Bayerische Julius-Maximilians-Universität Würzburg, Germany \\ E-mail: Philipp.Loesel@physik.uni-muenchen.de
}

Currently square-meter sized Micromegas detectors with a spatial resolution better than $100 \mu \mathrm{m}$ are of big interest for many experiments. Due to their size the construction of these detectors is highly sophisticated as they need to fulfill strict mechanical tolerances. We developed a method to survey working detectors on potential deviations of the micro pattern readout structures from design value as well as deformations of the whole detector, using cosmic muons in a tracking facility.

The LMU Cosmic Ray Facility consists of two $8 \mathrm{~m}^{2}$ Monitored Drift Tube chambers (ATLAS MDT) for precision muon tracking and two segmented trigger hodoscopes providing $10 \mathrm{~cm}$ position information along the wires of the MDTs with sub-ns time-resolution. It provides information on homogeneity in efficiency and pulse height of one or several Micromegas installed between the MDTs. With an angular acceptance of $-30^{\circ}$ to $+30^{\circ}$ the comparison of the MDT muon tracking with centroidal position determination or TPC-like track reconstruction in the Micromegas allows for calibration in three dimensions.

We currently investigate a detector system consisting of a $1 \mathrm{~m}^{2}$ and three $100 \mathrm{~cm}^{2}$ resistive strip Micromegas, with emphasis on the differences in performance between large and small detectors. The small detectors behave dimensionally stable, whereas the large detector seems to show deviations from the readout strip straightness and global deformation due to the small overpressure caused by the Ar: $\mathrm{CO}_{2}$ gas flux. We introduce the alignment and calibration procedure, report on homogeneity in efficiency and pulse height and present results on deformation and performance of the $1 \mathrm{~m}^{2}$ Micromegas. The same measurement will be performed with a 4-layer Micromegas quadruplet of $2 \mathrm{~m}^{2}$ size, in near future.

38th International Conference on High Energy Physics

3-10 August 2016

Chicago, USA

* Speaker. 


\section{Introduction}

Micro-Mesh Gas detectors (Micromegas) [1] are high precision gas detectors consisting of three planar structures, a cathode, a stainless steel micro mesh and a strip anode. A traversing charged particle ionizes the gas atoms along its path. The electrons drift through the mesh and create gas avalanches in the amplification region. The signals are detected on the readout strips by capacitive coupling from the resistive strips which are insulated via $50 \mu \mathrm{m}$ of Kapton (see fig. 1). The resistive strip technology is used as discharge protection [2]. Without discharge protection a streamer would equalize the potentials of mesh and anode. Hence no gas amplification would be possible during recharge time of the anode. The resistive strip technology prevents this on a global scale and reduces this to a tiny localized effect with negligible deadtime. Small Micromegas of about $100 \mathrm{~cm}^{2}$ in size can be built precisely and have thus an excellent spatial resolution as shown in [3].

\section{Experimental Setup}

The Cosmic Ray Facility (CRF) [4] at LMU consists of two $4 \times 2.2 \mathrm{~m}^{2}$ Monitored Drift Tube (MDT) chambers with six tube layers each (see fig. 2 (left)). Each provides a reference track in the precision direction $y$ which is used for the analysis of the test chamber. The MDTs are sandwiched by two trigger hodoscopes consisting of $10 \mathrm{~cm}$ wide scintillators, which provide a second coordinate $(x)$ along the wires of the drift tubes, hence allow for virtual segmentation of the test chamber in $10 \mathrm{~cm}$ wide segments. Between lower MDT and lower hodoscope a $34 \mathrm{~cm}$ thick iron absorber is placed hardening the muon spectrum to energies above $600 \mathrm{MeV}$. With its large area and an angular acceptance of $-30^{\circ}$ to $+30^{\circ}$ the CRF allows for investigation and calibration of square-meter sized detectors.

The test chamber, in this case a $92 \times 102 \mathrm{~cm}^{2}$ large Micromegas (L1), is placed between the MDTs with the readout strips parallel to the wires. The one-dimensional readout of the Micromegas gives position information perpendicular to the strips but is not position sensitive along the strips. Due to the large area of the Micromegas it consists of two readout boards which are glued together in the middle of the detector. It has a strip pitch of $450 \mu \mathrm{m}$, an amplification gap of $128 \mu \mathrm{m}$ and a drift gap of $5 \mathrm{~mm}$. A gas mixture of $\mathrm{Ar}: \mathrm{CO}_{2} 93: 7$ vol\% is used. The mesh is grounded and typical electrical fields are $E_{\text {drift }}=600 \mathrm{~V} / \mathrm{cm}$ and $E_{\text {ampl }}=44.5 \mathrm{kV} / \mathrm{cm} .16$ APV25 front-end boards [5] are used to readout this detector and together with the ten segments along the strips the chamber can be subdivided into 160 partitions. For each of these partitions the alignment and calibration is done separately (see fig. 2 (right)). 

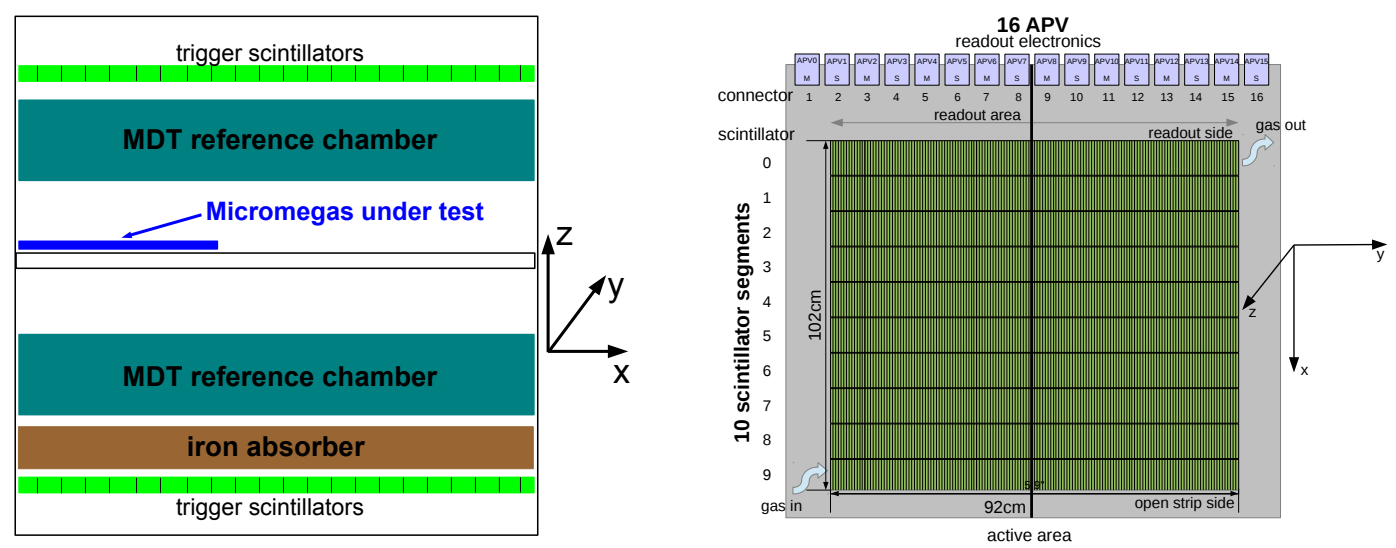

Figure 2: Schematic of the LMU Munich Cosmic Ray Facility (CRF) consisting of two Monitored Drift Tube chambers (MDTs) sandwiching the test Micromegas, two trigger hodoscopes providing a second coordinate along the wires and strips and a $34 \mathrm{~cm}$ iron absorber (left). Top view of the $1 \mathrm{~m}^{2}$ Micromegas under test, consisting of two readout boards. The Micromegas is read out by 16 APV25. The 16 APV25 and the 10 scintillator segments allow for a subdivision into 160 partitions (right).

\section{Precision Calibration Using Cosmic Muons}

For the calibration of the $1 \mathrm{~m}^{2}$ Micromegas the position of the traversing muon in the test chamber has to be reconstructed. This is done via the so-called centroid method. Here the strip position is weighted with the maximum charge on the strip and the average is built by $y_{\text {cen }}=$ $\sum_{\text {strips }} y_{\text {strip }} \cdot q_{\text {strip }} / \sum_{\text {strips }} q_{\text {strip }}$, where $y$ is the position and $q$ the charge. The difference of this position and the predicted position from the MDT reference track is called the residual $y_{\text {res. }}$. The residual as a function of the reference track slope $m_{y}$ leads immediately to $z$ and $y$ position of the test detector by linear

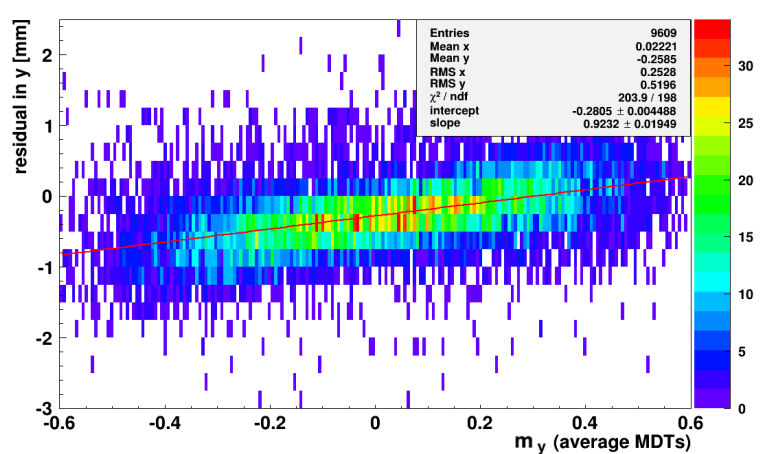

Figure 3: Difference of measured and predicted track position (residual) as a function of the reference track slope. The red line is a linear fit to the data where the slope is the $\mathrm{z}$ position and the intersection is the $\mathrm{y}$ position of the test detector.

fit, where the intersection gives the $y$ position $\Delta y=y_{\mathrm{res}}$ and the slope the $z$ position $\Delta z=\Delta y_{\mathrm{res}} / \Delta m_{y}$. This is done for all 160 partitions and leads to a 3D map of the Micromegas. After overall alignment with respect to the reference chambers all mechanical deformations can be identified using this calibration procedure as well.

\subsection{Deformation of the Drift Region}

Figure 4 (left) shows the position in $z$ for all 160 partitions. A mechanical deformation corresponding to the inflation of the detector by 10 mbar overpressure can be clearly seen. The centroid method allows to reconstruct positions in the middle of the drift gap with a resolution better than $100 \mu \mathrm{m}$. Therefore the measured deformation of about $0.7 \mathrm{~mm}$ in $\Delta \mathrm{z}$ is only half of the real de- 

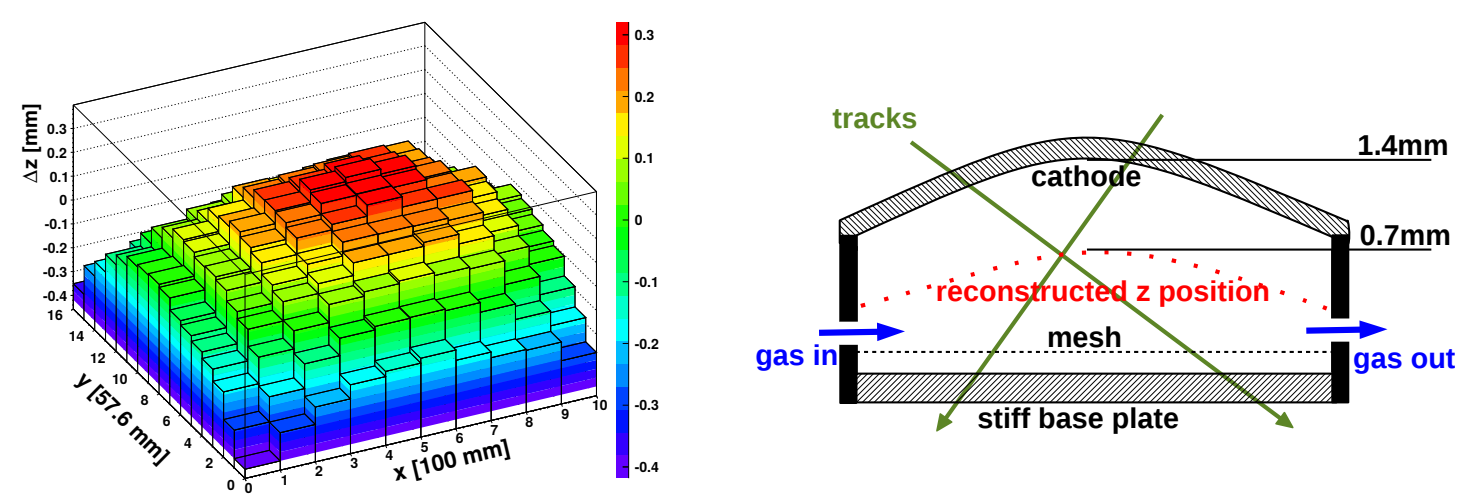

Figure 4: Measured deformation of the drift region (left) and scheme of the mechanical deformation due to 10 mbar overpressure (right). The analysis reconstructs the middle of the drift region.

formation of the detector. This deformation occurs due to the 10 mbar ram pressure caused by the flowing gas through the detector. The deformation is directed only upwards, because the base plate of the chamber is much stiffer than the reinforcement of the cathode (see fig. 4 (right)).

\subsection{Determination of the Average Strip Positions}

By plotting the residual in $y$ for all 160 partitions the two readout boards can be clearly distinguished (see fig. 5). The $\mathrm{z}$-axis shows at $y=8$ the displacement of the second readout board, when the first is aligned to the MDTs. A shift at the upper end of about $100 \mu \mathrm{m}$ and a rotation of about $350 \mu \mathrm{m} / \mathrm{m}$ can be seen. The determination of the strip positions in precision direction was measured with an accuracy of $15 \mu \mathrm{m}$. This displacement of the two readout boards occurs due to no alignment during the gluing

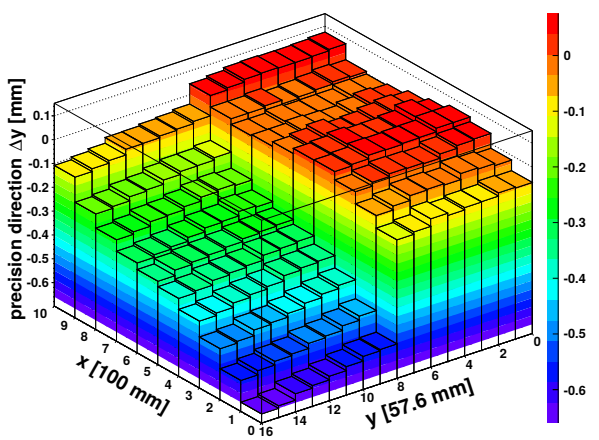

Figure 5: Measured residual in $y$ as a function of the position in the test Micromegas. A shift of $100 \mu \mathrm{m}$ and a rotation of $350 \mu \mathrm{m} / \mathrm{m}$ between the two readout boards is observed. procedure for this test chamber. But the chamber works well and this effect can be calibrated as given.

\section{Micromegas Telescope in the Cosmic Ray Facility}

To investigate the properties of the $1 \mathrm{~m}^{2}$ Micromegas (L1) further and compare the results with a smaller, well understood Micromegas, additional Micromegas detectors were installed in the CRF. These Micromegas form a telescope in between the MDTs consisting of the $1 \mathrm{~m}^{2}$ chamber, two $9 \times 9 \mathrm{~cm}^{2}$ Micromegas with two-dimensional readout (Tmm1 and Tmm2) and a $10 \times 10 \mathrm{~cm}^{2}$ Micromegas with one-dimensional readout (T1). The two-dimensional Micromegas with $250 \mu \mathrm{m}$ strip pitch provide track information in both directions on the order of $100 \mu \mathrm{m}$. For comparison to L1 the T1 chamber with one-dimensional readout is used, which has a similar design and strip pitch of $400 \mu \mathrm{m}$. Each detector was individually aligned to the MDTs. 
To determine the influence of multiple scattering on the spatial resolution the residual distributions are fitted with a double Gaussian function to extract the spatial resolution. In this analysis the track resolution was not considered yet. Hence in the following the weighted standard deviations of the double Gaussian function will be referred to as spatial resolution. This is done as a function of the reference angle and for different cuts on the slope difference between the two MDT tracks (see fig. 7 (left)). Low energy muons scatter more when traversing matter, this leads to larger differences between the two reference tracks. It can be clearly seen that the spatial resolution worsens with a looser cut on the slope difference. In figure 7 (right) the spatial resolution

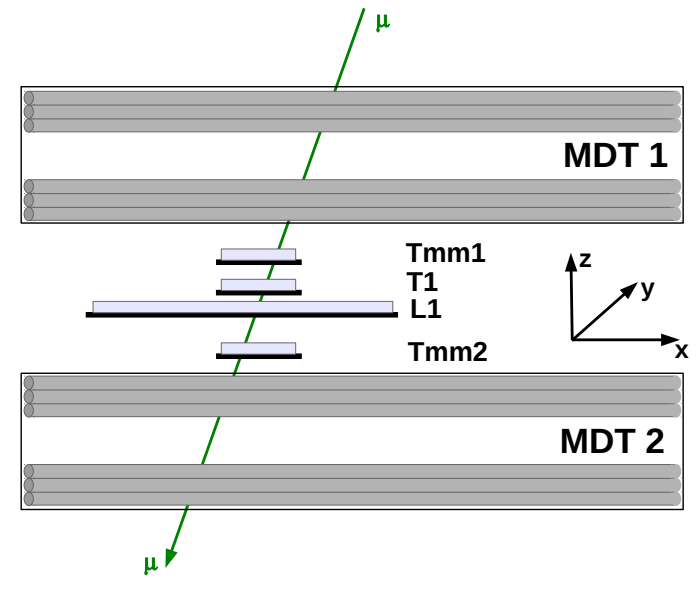

Figure 6: Scheme of the Micromegas Telescope installed in the CRF. Additional to the former setup two $9 \times 9 \mathrm{~cm}^{2}$ (Tmm1 and Tmm2) and one $10 \times 10 \mathrm{~cm}^{2}$ (T1) Micromegas were installed. for the large (L1) and small (T1) Micromegas is plotted as a function of the reference track inclination. In this case no cuts on multiple scattering were applied due to non sufficient statistics for the small detector. Additionally the spatial resolution for the area of L1 covered by the telescope was plotted. This is very similar to the resolution of the small detector, whereas the resolution for the whole L1 chamber is slightly worse. For this the calibration can still be improved.
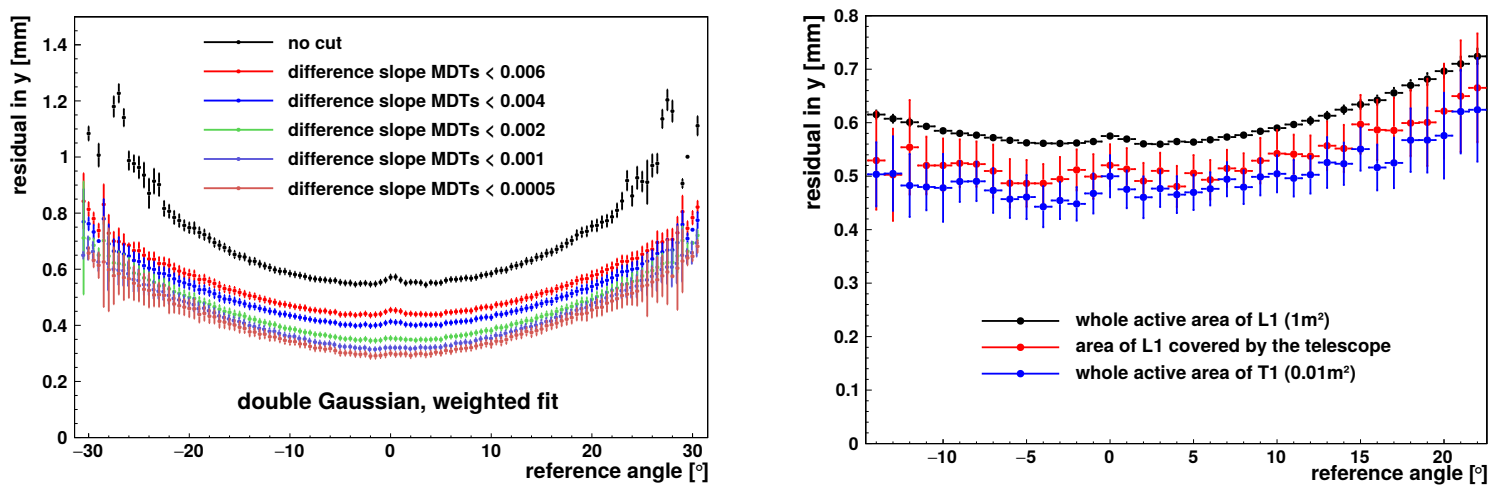

Figure 7: Spatial resolution of the large Micromegas L1 as a function of the reference track angle for different cuts on the difference of the slope of the two reference tracks (left). The residual distribution for each angle is fitted with a double Gaussian function and the standard deviations are weighted by the integral. Spatial resolution as a function of the reference track angle for the large (L1) and small (T1) Micromegas and for the part of the large Micromegas covered by the telescope (right).

\section{Homogeneity in Efficiency and Pulse Height}

The $3 \sigma$ efficiency $\epsilon_{3 \sigma}$ is defined by the number of reconstructed tracks within $\pm 3 \sigma$ around the expected position by the total number of tracks. This was calculated for all 160 partitions of the calibration grid (see 8 (left)). The detector shows a good homogeneity with $\overline{\epsilon_{3 \sigma}}=(92 \pm 1) \%$. The efficiency as well as the spatial resolution is limited by multiple scattering. This can be seen 
more pronounced with the Micromegas telescope installed. Here the detectors show a lower $3 \sigma$ efficiency when more material is in the path of the muons (see 8 (right)).

The large detector has also shown a homogeneous gas gain distribution within $20 \%$.
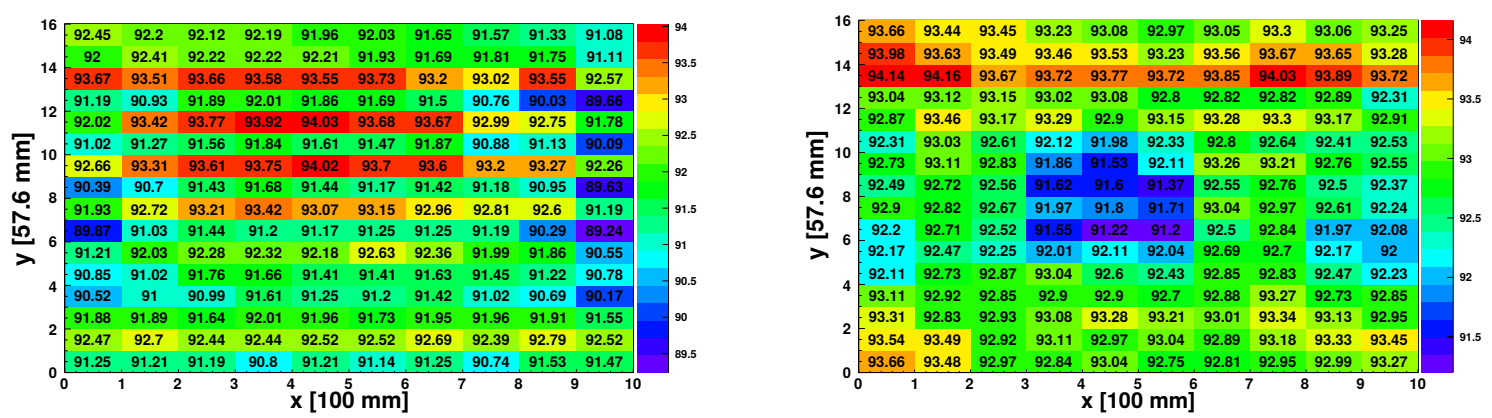

Figure 8: $3 \sigma$ efficiency for each of the 160 partitions of the calibration without telescope (left) and with installed telescope (right). A lower $3 \sigma$ efficiency is visible in the area of the telescope (blue at $x=3-6$ ) due to increased multiple scattering.

\section{Summary}

We have presented a calibration method for large area micro-pattern gas detectors using cosmic muons. Mechanical deformations in the drift region and the displacement between readout boards of a $1 \mathrm{~m}^{2}$ Micromegas detector have been located with high precision in the LMU Cosmic Ray Facility. After calibration a $1 \mathrm{~m}^{2}$ Micromegas shows similar performance as a $10 \times 10 \mathrm{~cm}^{2}$ detector. Not considering the track resolution, in both cases the achievable spatial resolution is limited by multiple scattering of the cosmic muons. It also has been shown that the resolution for a small part of the $1 \mathrm{~m}^{2}$ is better than for the whole chamber, thus the alignment can be further improved. The large detector shows a good homogeneity in pulse height and efficiency. The multiple scattering is more pronounced in areas with more material in the muon path which can be seen in the $3 \sigma$ efficiency.

\section{Acknowledgments}

We acknowledge the support by the DFG Excellence Cluster 'Origin and Structure of the Universe'.

\section{References}

[1] Y. Giomataris et al., Micromegas: a high-granularity position sensitive gaseous detector for high particle-flux environments, Nucl. Instr. Meth. A 37629 - 35 (1996).

[2] T. Alexopoulos et al., A spark-resistant bulk-Micromegas chamber for high-rate applications, Nucl. Instrum. Meth. A 640110 (2011) [arXiv:1011.5370].

[3] J. Manjarres et al., Performances of Anode-resistive Micromegas for HL-LHC, JINST 7 C03040 (2012) [arXiv:1202.1074].

[4] J. Dubbert et al., First Results from the Cosmic-Ray Test-Facility at LMU Munich, http://cds.cern.ch/record/681507/files/muon-2005-008.pdf (2002).

[5] L. Jones, APV25-S1 User Guide, http://cds.cern.ch/record/1069892/files/ cer-002725643.pdf (2001). 